\title{
Publisher Correction: Artificial metalloenzymes: Reversible catalyst anchoring
}

Jun Okuda

Correction to: Nature Catalysis https://doi.org/10.1038/s41929-018-0149-7, published online 12 September 2018.

In the version of this News \& Views article originally published, in ref. 3, 'https://doi.org/10.1038/s41929-018-124-3' was incorrect and should have read 'https://doi.org/10.1038/s41929-018-0124-3'. This has now been corrected in all versions. 